\title{
Human epithelial cells trigger dendritic cell-mediated allergic inflammation by producing TSLP
}

Vassili Soumelis', Pedro A. Reche', Holger Kanzler', Wei Yuan', Gina Edward', Bernhart Homey',2, Michel Gilliet', Steve Ho', Svetlana Antonenko', Annti Lauerma33, Kathleen Smith', Daniel Gorman', Sandra Zurawski', Jon Abrams', Satish Menon', Terri McClanahan', Rene de Waal-Malefyt', Fernando Bazan', Robert A. Kastelein' and Yong-Jun Liu'

Whether epithelial cells play a role in triggering the immune cascade leading to $T$ helper $2\left(T_{H} 2\right)$-type allergic inflammation is not known. We show here that human thymic stromal lymphopoietin (TSLP) potently activated $\mathrm{CDI} / \mathrm{c}^{+}$dendritic cells $(\mathrm{DCs})$ and induced production of the $\mathrm{T}_{\mathrm{H}} 2$-attracting chemokines TARC (thymus and activation-regulated chemokine; also known as CCLI7) and MDC (macrophage-derived chemokine; CCL22).TSLP-activated DCs primed naïve $T_{H}$ cells to produce the proallergic cytokines interleukin 4 (IL-4), IL-5, IL-I3 and tumor necrosis factor- $\alpha$, while downregulating IL-IO and interferon- $\gamma$. TSLP was highly expressed by epithelial cells, especially keratinocytes from patients with atopic dermatitis. TSLP expression was associated with Langerhans cell migration and activation in situ. These findings shed new light on the function of human TSLP and the role played by epithelial cells and DCs in initiating allergic inflammation.

About $20 \%$ of the population in Western countries suffers from allergic diseases, which include asthma, allergic rhinitis, atopic dermatitis and food allergy ${ }^{1}$. Allergic inflammation is the result of a complex immunological cascade that leads to dysregulated production of $\mathrm{T}$ helper type $2\left(\mathrm{~T}_{\mathrm{H}} 2\right)$-derived cytokines such as interleukin 4 (IL-4), IL5 and $\mathrm{IL}-13^{2-4}$, which trigger immunoglobulin $\mathrm{E}$ ( $\mathrm{IgE}$ ) production, eosinophilia and mucus production ${ }^{5-7}$. Dendritic cells (DCs), which are professional antigen-presenting cells ${ }^{8}$, play an important role in the pathogenesis of allergic diseases ${ }^{9-11}$. However, the initial signal that primes DCs to induce $\mathrm{T}$ cells to produce proallergic $\mathrm{T}_{\mathrm{H}} 2$ cytokines is unknown. Epithelial cells are located at the sites of allergen entry into the body and interact closely with DCs in situ. However, it is not known whether DCs play a role in triggering the allergic immune cascade. Although skin keratinocytes and mucosal epithelial cells produce proinflammatory cytokines such as IL-1, IL6, IL-8, granulocyte-macrophage colony-stimulating factor (GM$\mathrm{CSF}$ ) and tumor necrosis factor- $\alpha$ (TNF- $\alpha)$ after activation ${ }^{12}$, none of these cytokines explain the mechanism that underlies the induction of allergic inflammation.

Thymic stromal lymphopoietin (TSLP) is an IL-7-like cytokine, cloned from a murine thymic stromal cell line ${ }^{13}$. The TLSP receptor is a heterodimer that consists of the IL-7 receptor $\alpha$ chain (IL-7R $\alpha$ ) and a common $\gamma$-like receptor chain called TSLP receptor (TSLPR $)^{14-17}$ Mouse TSLP supports murine early B and T cell developments ${ }^{18,19}$ and does not appear to have any biological effects on murine DCs (unpublished data). In contrast, human TSLP activates CD11 $\mathrm{c}^{+}$DCs, but does not appear to have any direct biological effects on B cells, T cells, NK cells, neutrophils or mast cells ${ }^{17}$. This is in accordance with the coexpression of IL-7R $\alpha$ chain and TSLPR mRNA in CD11 $\mathrm{c}^{+}$DCs, but not in other cell types. We show here that human TSLP potently activated human $C D 11 \mathrm{c}^{+} \mathrm{DCs}$, which subsequently primed naïve $\mathrm{T}_{\mathrm{H}}$ cells to produce high concentrations of IL-13, IL-5 and TNF- $\alpha$, moderate amounts of IL-4 and down-regulate IL-10 and interferon- $\gamma$ (IFN- $\gamma$ ). TSLP is highly expressed by epithelial cells of inflamed tonsils and keratinocytes of atopic dermatitis. Thus, TSLP represents a key epithelial cell or keratinocyte-derived cytokine that directly triggers DC-mediated allergic inflammation.

\section{Results}

TSLP potently activates human CDI I c ${ }^{+}$DCs

We compared the effects of TSLP, IL-7, CD40 ligand (CD40L)and lipopolysaccharide (LPS) on human CD11 $\mathrm{c}^{+}$DC activation. TSLP, IL-7, CD40L and LPS all up-regulated surface HLA-DR, CD40, CD80, CD86 and CD83 on DCs when compared with medium alone (Fig. 1a). Whereas TSLP induced the most CD40 and CD80 expression on DCs, CD40L induced more HLA-DR and CD83. Like CD40L, TSLP not only activated DCs, but also maintained the survival of DCs in 24-h cultures, as shown by annexin V staining (Fig. 1a) and cell counts (data not shown). 
The ability of TSLP to up-regulate HLA-DR and costimulatory molecules was blocked by two neutralizing monoclonal antibodies (mAbs) - 5E5 and $12 \mathrm{~F} 3$ - that were specific for human TSLP (Fig. 1b). The two TSLP mAbs did not block the up-regulation of HLA-DR and costimulatory molecules on DCs induced by CD40L, IL-7 or LPS (data not shown). These data indicated that the observed effects of TSLP on CD11 $\mathrm{c}^{+}$DCs were TSLP-specific. Morphologically, both TSLP-activated DCs (referred to hereafter as TSLP-DCs) and CD40L-activated DCs (referred to hereafter as CD40L-DCs) showed long dendrites and expressed more HLA-DR and DC-lysosome-associated membrane protein (DC-LAMP, which is a DC activation marker) compared to DCs in medium alone or IL-7-activated DCs (referred to hereafter as IL-7-DCs) (Fig. 1c).

a
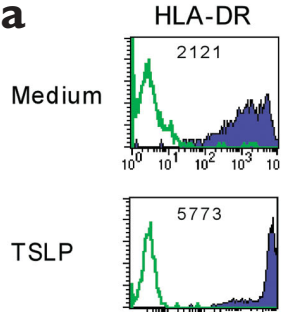

IL-7

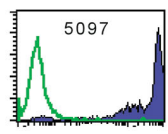

CD40L

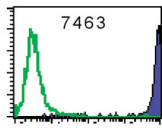

LPS

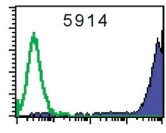

b
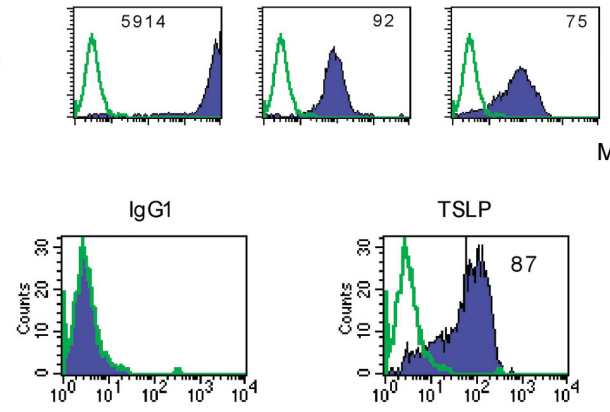

MF
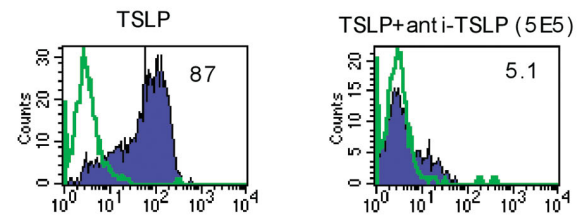

MFI
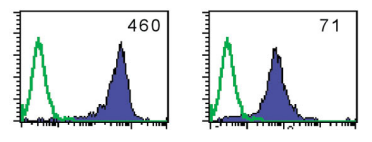

\section{C}
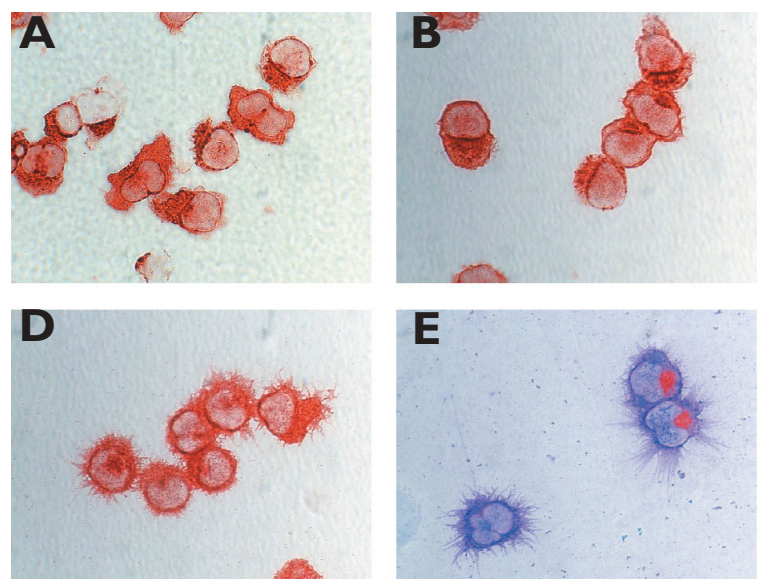

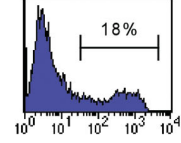

TSLP-DCs induce CD4 ${ }^{+} \mathrm{T}$ cell expansion

Compared to CD40L-DCs, LPS-activated DCs (referred to hereafter as LPS-DCs) or IL-7-DCs, TSLP-DCs induced stronger naïve CD4+ $\mathrm{T}$ cell proliferation in an allogeneic mixed lymphocyte reaction (Fig. 2a). At a 1:150 ratio of DCs:T cells, TSLP-DCs still induced allogeneic naïve $\mathrm{CD}^{+}{ }^{+} \mathrm{T}$ cell proliferation that was about three-times stronger than that induced by CD40L-DCs (Fig. 2a). After 6 days of culture, TSLPDCs induced a 7.5- to 9-fold increase in total $\mathrm{T}$ cell numbers, which was more than that induced by CD40L-DCs, LPS-DCs or IL-7-DCs (Fig. 2b). Therefore, human TSLP represents one of the most potent DC activation factors, and TSLP-DCs induce the most marked allogeneic naïve $\mathrm{CD} 4^{+} \mathrm{T}$ cell proliferation and expansion.
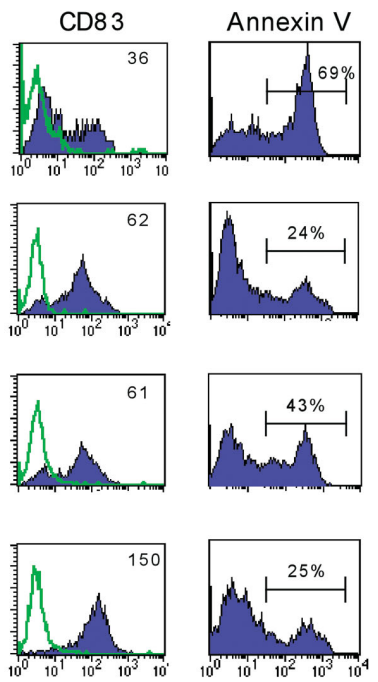

Figure I.TSLP potently activates CDI Ic ${ }^{+}$DCs and maintains their survival. (a) TSLP strongly up-regulates HLA-DR, CD40, CD80, CD86 and CD83 compared to medium alone, and it potently up-regulates CD40 and CD80 expression compared to other DC activators (CD40L, IL-7 and LPS). Filled histograms represent staining of DC activation markers; open histograms represent the isotype control. Numbers indicate the mean fluorescence intensity (MFI). The percentage of annexin $\mathrm{V}^{+}$apoptotic DCs was also greatly reduced after $24 \mathrm{~h}$ of culture with TSLP (24\%), CD40L (25\%) and LPS (I8\%) compared with those cultured with medium (69\%) or IL-7 (43\%) Data represent one of six independent experiments. (b) Surface CD80 expression by DCs is strongly induced by TSLP, which is specifically blocked by rat anti-TSLP (mAbs 5E5 and I2F3). Numbers indicate the MFI for CD80 expression. Data represent one of three independent experiments. (c) Morphological analysis of DCs on cytospins. Major histocompatibility complex (MHC) class II staining of CDII c ${ }^{+}$DCs cultured with $(\mathbf{A})$ medium (B) IL-7 (C) CD40L or (D) TSLP. MHC class II (blue) and DC-LAMP (red) double-staining of DCs cultured with (E) CD40L or (F) TSLP. 

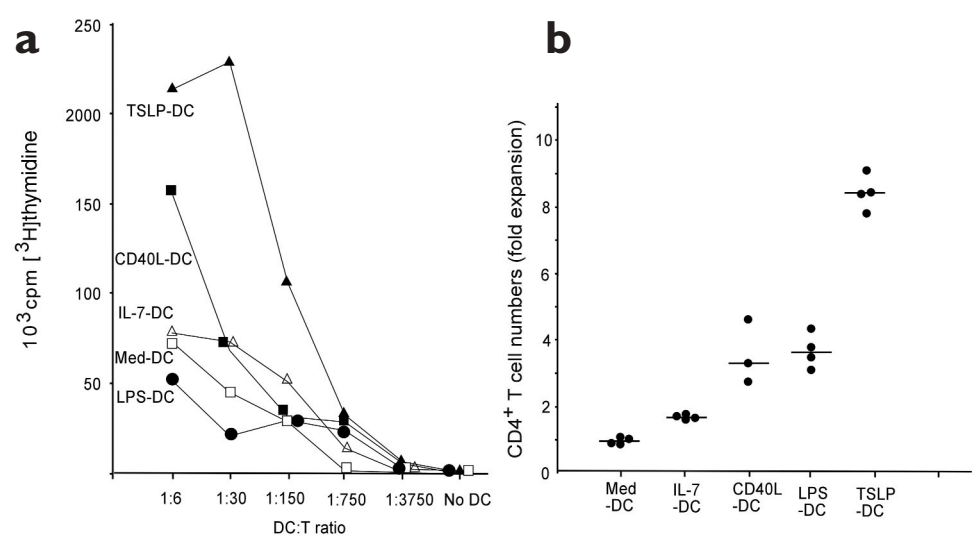

Figure 2. Naïve $C D 4^{+} \mathrm{T}$ cell proliferation and expansion with DCs activated by TSLP and other activators. (a) TSLP-DCs induced the strongest $C D 4^{+} \mathrm{T}$ cell proliferation after 5 days of culture, as assessed by $\left[{ }^{3} \mathrm{H}\right]$ thymidine incorporation both at high $(\mathrm{I}: 6)$ and low (I:I50) DC:T ratios. (b) TSLP-DCs induced the highest CD4 $4^{+} \mathrm{T}$ cell expansion after 6 days of culture. Results are expressed as fold expansion compared to the initial T cell number (50,000 cells). Data represent five independent experiments; horizontal bars indicate the median.

\section{TSLP-DCs induce $T_{\mathrm{H}} \mathbf{2}$ development}

Most DC activation signals, such as CD40L and LPS, induce DCs to produce the proinflammatory cytokines IL- $1 \alpha / \beta$, IL- 6 and IL-12 20,21 and prime naïve $\mathrm{CD} 4^{+} \mathrm{T}$ cell differentiation towards $\mathrm{T}_{\mathrm{H}} 1^{22-24}$. We performed a global quantitative mRNA screening of 11 different cytokines-IL- $1 \alpha$, IL-1 $\beta$, IL-4, IL-6, IL-10, IL-12p35, IL-12p40, IL-13, IL-18, IL-23p19 and TNF- $\alpha$ - and 12 different chemokines - thymus and activation-regulated chemokine (TARC also known as CCL17), DCCK1, macrophage-derived chemokine (MDC or CCL22), monocyte chemoattractant protein 1 (MCP-1 or CCL2), MCP-2 (or CCL8), MCP-3 (or CCL7), MCP-4 (or CCL13), eotaxin (or CCL11), macrophage inflammatory protein (MIP-3 $\beta$ or CCL19), monokine induced by $\gamma$ interferon (MIG or CXCL9), RANTES (or CCL5) and IL-8 (or CXCL8) - which have potential effects either on naïve $C D 4^{+} \mathrm{T}$ cell polarization or the migration of $\mathrm{T}_{\mathrm{H}} 1$ or $\mathrm{T}_{\mathrm{H}} 2$ cells. Unlike CD40L-DCs and LPS-DCs, TSLP-DCs did not produce mRNA for all the proinflammatory cytokines tested, but did produce high levels of mRNA for the chemokines TARC and MDC (data not shown). Enzyme-linked immunosorbent assay (ELISA) analyses confirmed at the protein level that TLSP-DCs did not produce detectable amounts of the proinflammatory cytokines IL- $1 \beta$, IL-6, IL-12p70 and TNF- $\alpha$, but did produce high amounts of the chemokines TARC and MDC (Fig. 3). TARC and MDC preferentially attract CCR4-expressing $\mathrm{T}_{\mathrm{H}} 2$ cells ${ }^{25}$.

The capacity of TSLP-DCs to polarize naïve $\mathrm{CD}^{+} \mathrm{T}$ cells was compared to DCs cultured with medium, IL-7, CD40L or LPS. Naïve human $\mathrm{CD}^{+}{ }^{+} \mathrm{CD} 45 \mathrm{RA}^{+} \mathrm{T}$ cells purified from adult peripheral blood were cul- tured with DCs at a 1:5 ratio for 6 days; they were then washed to remove all cytokines, restimulated for $24 \mathrm{~h}$ with anti-CD3 and antiCD28 and then cytokine production was measured in the culture supernatant by ELISA. TSLP-DCs induce naïve $\mathrm{CD}^{+} \mathrm{T}$ cells to produce large amounts of IL-13, IL-5 and TNF- $\alpha$ and a moderate amount of IL-4 (Fig. 4a). Compared to DCs cultured with medium alone or other activators, TSLP-DCs induced naïve CD4 ${ }^{+} \mathrm{T}$ cells to produce the lowest amounts of the anti-inflammatory cytokine IL-10 and the $\mathrm{T}_{\mathrm{H}} 1$ cytokine IFN- $\gamma$ (Fig. 4a). The ability of TSLP-DCs to induce naïve CD4+ $\mathrm{T}$ cells to produce high IL-13 and TNF- $\alpha$, moderate IL- 4 and low IFN- $\gamma$ and IL-10 was confirmed by intracellular cytokine staining (Fig. $\mathbf{4 b}$ ). Therefore, TSLP-DCs induced naïve $C D 4^{+} \mathrm{T}$ cells to produce a unique set of cytokines that was distinct from a $\mathrm{T}_{\mathrm{H}} 1$ profile (IFN- $\gamma$ ) or a classical $\mathrm{T}_{\mathrm{H}} 2$ profile (IL-4, IL- 5 and IL-10). Compared with CD4 ${ }^{+}$T cells activated by DCs cultured in medium, IL-7-DCs, CD40L-DCs or LPS-DCs, CD4+ T cells primed with TSLP-DCs produced the highest amounts of TNF- $\alpha$, one of the most potent proinflammatory cytokines. In contrast, TSLPDCs inhibited IL-10 as well as IFN- $\gamma$ production by $\mathrm{CD}^{+} \mathrm{T}$ cells.

Therefore, TSLP-DCs may induce robust $\mathrm{T}_{\mathrm{H}} 2$ allergic inflammation by inducing naïve $\mathrm{CD} 4^{+} \mathrm{T}$ cells to produce large amounts of IL-13 and IL- 5 and a moderate amount of IL- 4 in the presence of TNF- $\alpha$ and in the absence of two physiologic inhibitors of $\mathrm{T}_{\mathrm{H}} 2$ inflammation, IL-10 and IFN- $\boldsymbol{\gamma}^{26,27}$. In addition, TSLP-DCs may further enhance $\mathrm{T}_{\mathrm{H}} 2$-mediated inflammation by producing chemokines such as TARC and MDC, which may preferentially recruit $\mathrm{T}_{\mathrm{H}} 2$ cells into the original inflamed tissues ${ }^{28-30}$.

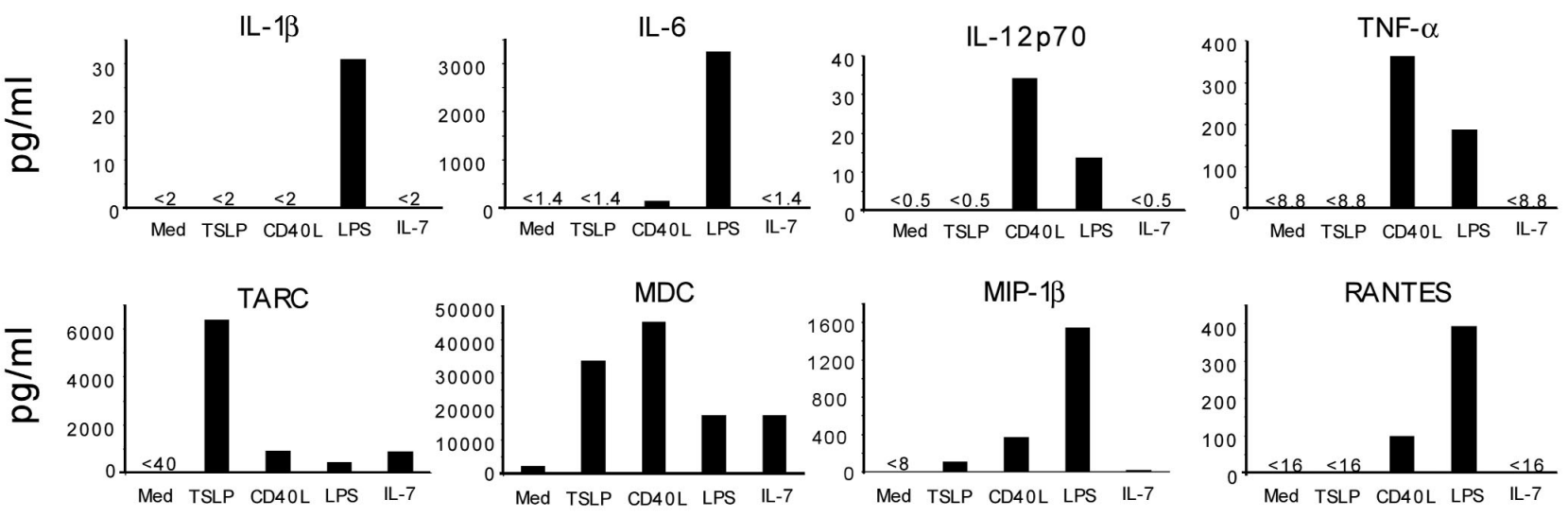

Figure 3. Cytokine and chemokine production by DCs activated with TSLP. TSLP-DCs did not produce inflammatory cytokines compared to CD40L-DCs or LPSDCs, but produce high amounts of the $\mathrm{T}_{\mathrm{H}} 2$-attracting chemokines TARC and MDC. Data represent one of five experiments. 
a

IL4
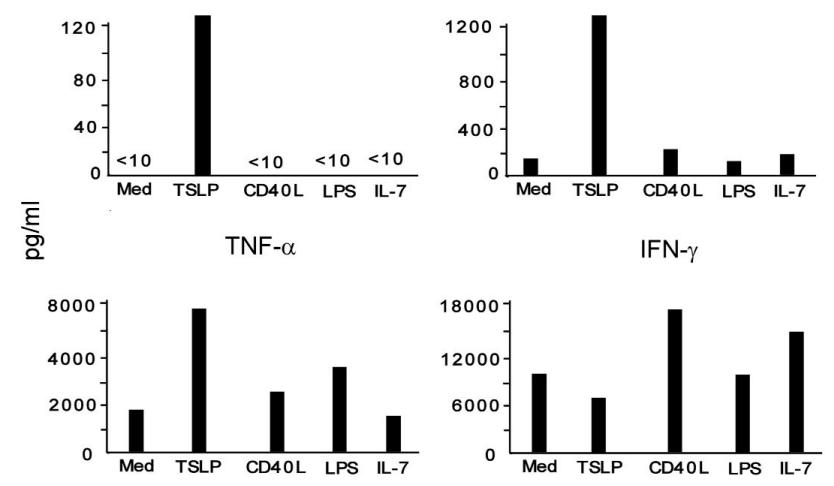

IFN- $\gamma$

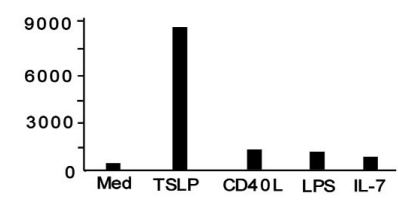

IL-10

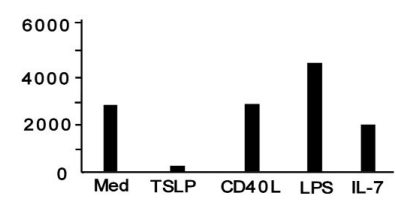

b
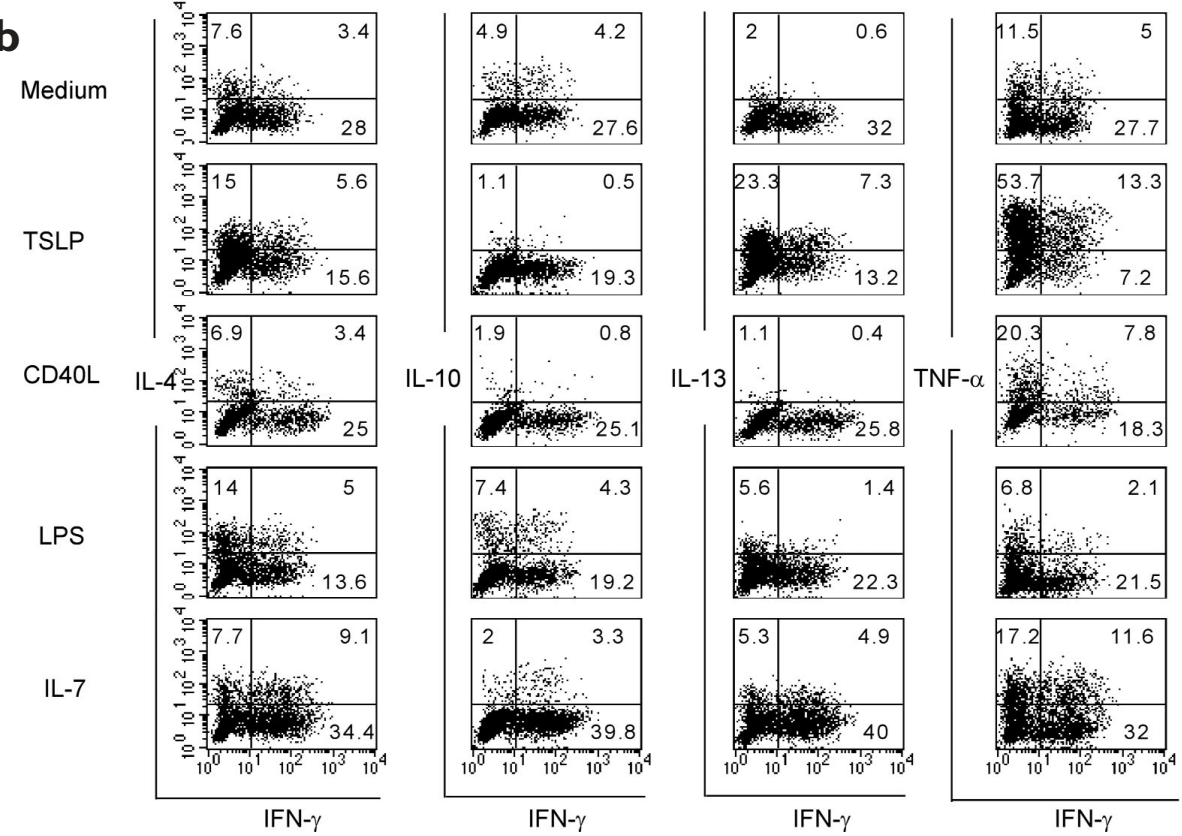

Figure 4. Cytokine production by naïve $\mathrm{CD4}^{+} \mathrm{T}$ cells primed for 6 days with TSLP-DCs. (a) TSLP-DCs prime CD4 $4^{+} \mathrm{T}$ cells to produce the highest amounts of IL4, IL-5, IL- 13 and TNF- $\alpha$, but lower amounts of IFN- $\gamma$ and IL-10, compared to mediumactivated DCs, CD40L-DCs, LPS-DCs or IL7-DCs. Data represent one of six independent experiments. (b) Intracellular cytokine staining of $T$ cells after 5 -h restimulation with PMA and ionomycin shows similar results. Data represent one of three independent experiments.

TSLP protein expression by epithelial cells

Human tonsils contain two types of epithelium: crypt epithelium, which frequently harbors viruses and bacteria and represents the site of antigenentry and constitutive inflammation, and squamous epithelium. Using the mAb $12 \mathrm{~F} 3$, we showed that TSLP is expressed by crypt epithelial cells, which were in close contact with cells expressing DC-LAMP in five different tonsillar samples tested ${ }^{31}$ (Fig. 6). In all tonsil samples tested, only a few small foci of TSLP expression were found within the apical part of the squamous epithelium (Fig. 7). The expression of TSLP was associated with the infiltration of DCLAMP $^{+}$-activated DCs (Fig. 7a,b) and the concurrent loss of langerin ${ }^{+}$ Langerhans cells within the squamous epithelium (Fig. 7c,d). Staining with $12 \mathrm{~F} 3$ was specific for TSLP because recombinant TSLP, but not IL-7, completely blocked the staining and rat Ig isotype control antibody did not give any positive staining

\section{TSLP mRNA expression}

To further understand the biology and pathophysiology of TSLP, expression of TSLP mRNA was analyzed by real-time quantitative polymerase chain reaction (PCR) in a panel of cDNA libraries from different cells or cell lines and a panel of purified primary cells (cell purity $>99 \%$ ) (Fig. 5). TSLP expression was not found in most hematopoietic cell types, including B cells, T cells, NK cells, granulocytes, macrophages, monocyte subsets and DC subsets; the exception was mast cells. Mast cells activated by mAbs that cross-link high-affinity IgE receptors express high amounts of TSLP. Human primary nonhematopoietic cells, such as skin keratinocytes, epithelial cells, smooth muscle cells and lung fibroblasts, cultured in growth medium, also expressed TSLP at high amounts, suggesting that these cells have the ability to produce TSLP (Fig. 5). Compared to cells cultured in medium, bronchial smooth muscle cells and skin keratinocytes activated by IL- 4 , IL-13 and TNF- $\alpha$ or TNF- $\alpha$ and IL- $1 \beta$ expressed higher TSLP. TSLP expression was not found in endothelial cells. Therefore, TSLP mRNA was mainly expressed by stromal cells, epithelial cells and mast cells, but not other hematopoietic cell types or endothelial cells. (data not shown). These results suggested that TSLP may contribute to constitutive inflammation within the crypt epithelium and sporadic inflammation within the squamous epithelium.

\section{High TSLP expression in atopic dermatitis}

To investigate whether TSLP expression is associated with $\mathrm{T}_{\mathrm{H}} 2$-type allergic inflammation in vivo, TSLP protein expression was analyzed in skin lesions, including atopic dermatitis $\left(\mathrm{a} \mathrm{T}_{\mathrm{H}} 2\right.$-mediated allergic disease), nickel-induced contact dermatitis (an IFN- $\gamma$-producing $\mathrm{T}$ cell-mediated allergic disease) and cutaneous lupus erythematosus samples. Although TSLP was undetectable in normal skin (11 separate samples) (Fig. 8a), high expression of TSLP was found in the keratinocytes of acute (ten patients, Fig. 8b,c) and chronic atopic dermatitis (five patients, Fig. 8d-f). Isotype control-staining of an adjacent section (as shown in Fig. 8d) gave a negative result. Expression of TSLP was found mainly in keratinocytes of the apical layers of the epidermis, which ranged from small foci to the whole apical areas in both acute and chronic atopic dermatitis. The characteristics of these patients with atopic dermatitis are summarized (Table 1). 


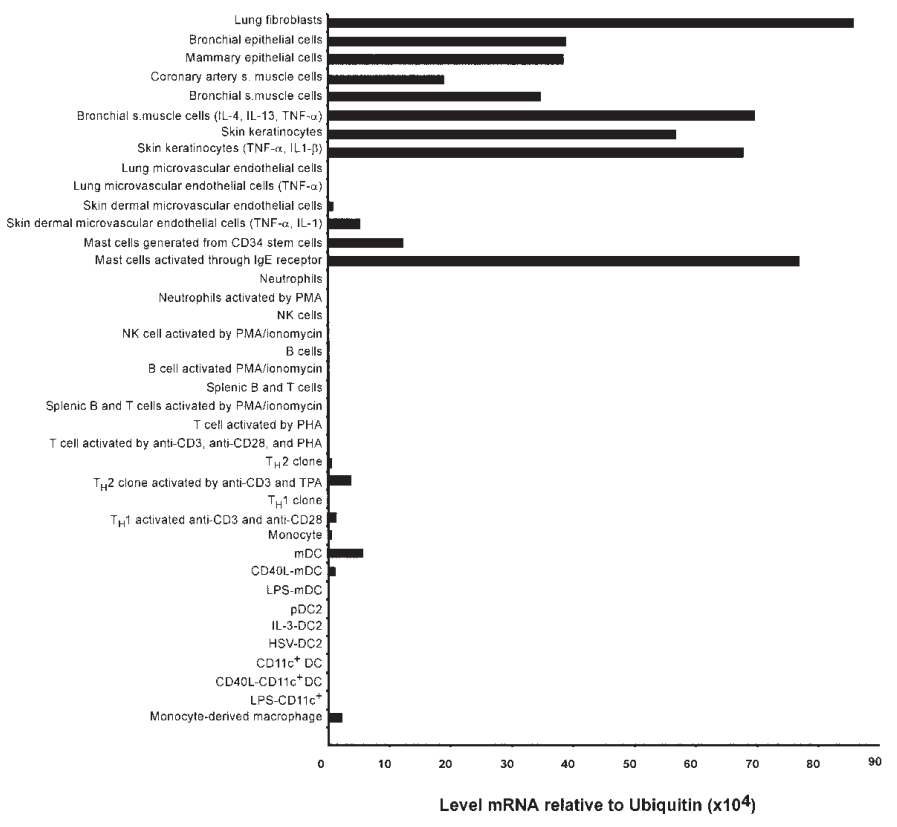

Figure 5. Quantification of TSLP mRNA levels in different human hematopoietic and stromal cell types. High levels of TSLP mRNA were detected in epithelial cells, different types of stromal cells and mast cells, but not in other hematopoietic cells.

Nonlesional skin samples were available for 9 of 15 atopic dermatitis patients. None of these samples stained positive for TSLP, which confirmed the specificity of TSLP for atopic dermatitis lesions and its absence from normal skin. Using Fisher's exact test, we were able to show that the difference between the normal skin group and atopic dermatitis group was statistically significant $(P<0.001)$. TSLP was not found in skin lesions from nickel-induced allergy contact dermatitis (Fig. 8h) or cutaneous lupus erythematosus (Fig. 8i) patients.

\section{TSLP associates with Langerhans cell activation}

To investigate whether TSLP expression in atopic dermatitis is associated with DC activation, TSLP was stained together with either langerin (the Langerhans cell marker) or DC-LAMP (the DC activation marker) in double immunohistology experiments (Fig. 9). In normal
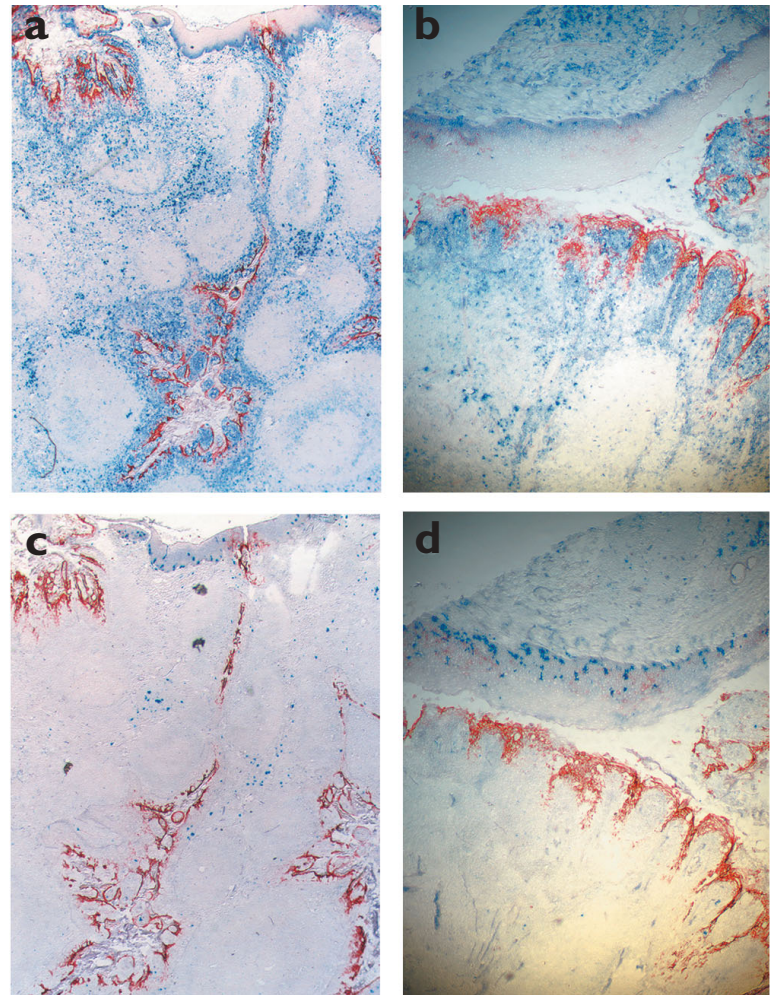

Figure 6. Expression of TSLP by crypt epithelial cells of human inflamed tonsils. (a,b) Double staining of TSLP (red) and DC-LAMP (blue). Expression of TSLP by crypt epithelial cells (red), which were in close association with DC-LAMP+ lymphocytes and DCs (blue). (a) Magnification: $\times 100$ (b) magnification: $\times 200$. (c,d) Double staining of TSLP (red) and langerin (blue). TSLP expression (red) by crypt epithelial cells, but not by squamous epithelial cells characterized by the presence of langerin+ Langerhans cells (blue). Langerin+ Langerhans cells within the epidermis do not express DC-LAMP. (c) Magnification: $\times 100$; (d) magnification: $\times 200$.

skin, or nonlesional skin of from atopic dermatitis samples, many langerin $^{+}$Langerhans cells were found within the epidermis, but not within the dermis (Fig. 9a); and no DC-LAMP+ activated DCs were found in either the epidermis or the dermis (Fig. 9b). Strong TSLP expression in atopic dermatitis was associated with the disappearance of langerin ${ }^{+}$
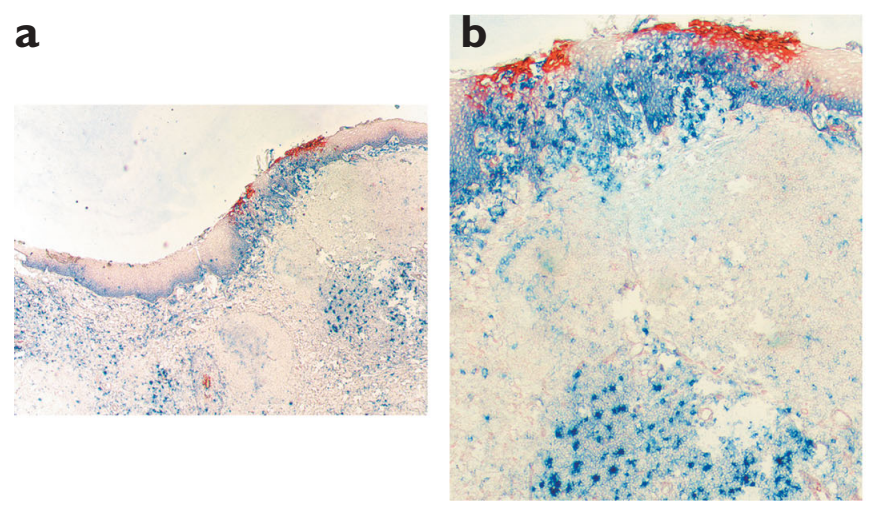

C

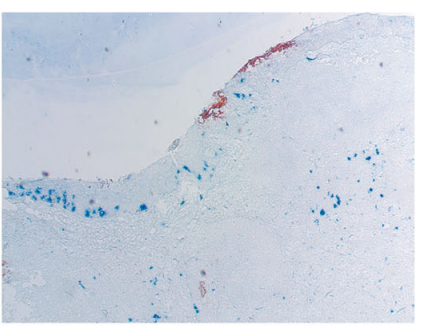

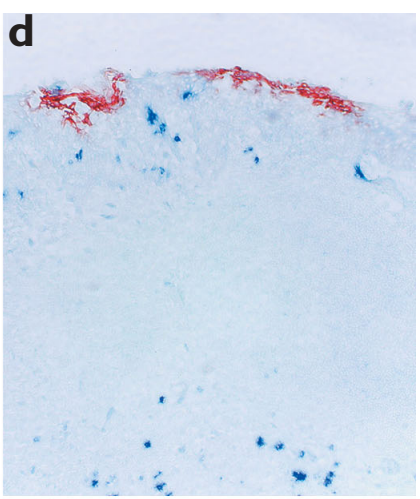

Figure 7. Sporadic expression of TSLP by squamous epithelial cells of inflamed tonsils is associated with the presence of activated DC-LAMP+ DCs. $(\mathbf{a}, \mathbf{b})$ Double staining with TSLP (red) and DC-LAMP (blue), showing DC-LAMP+ DCs within the epithelium area, which is positive for TSLP. (c,d) Double staining of TSLP (red) and langerin (blue), showing a decreased number of langerin ${ }^{+}$Langerhans cells within the TSLP-expressing epithelium. 
Langerhans cells within the epidermis (Fig. 9c) and the concurrent appearance of many DC-LAMP ${ }^{+}$activated DCs within the dermis (Fig. 9d). Many of the DC-LAMP $^{+}$activated DCs within the dermis expressed langerin, suggesting that epidermal Langerhans cells may be activated and migrate into the dermis (Fig. 9d). These results suggested that TSLP expression by keratinocytes in atopic dermatitis lesions may contribute directly to the activation of Langerhans cells, which may then migrate into the draining lymph nodes and prime allergen-specific $\mathrm{T}_{\mathrm{H}} 2$ responses.

\section{Discussion}

We have shown here that the epithelial cells of skin and mucosa may directly interact with DCs during allergic inflammation by producing TSLP. Human TSLP is a DC activator that displays several unique features compared with other DC activation factors, such as CD40L, LPS or IL-7. It induced more CD40 and CD80 expression on DCs compared to other activators, activated DCs to induce strong naïve $\mathrm{CD} 4^{+} \mathrm{T}$ cell proliferation and expansion, did not induce DCs to produce proinflammatory cytokines (instead it produced the $\mathrm{T}_{\mathrm{H}} 2$-attracting chemokines TARC and MDC) and it endowed DCs with the ability to prime naïve $\mathrm{CD} 4{ }^{+} \mathrm{T}$ cells to produce large amounts of IL-13, IL-5 and TNF- $\alpha$ and moderate amounts of IL-4. However, expression of the anti-inflammatory cytokine IL-10
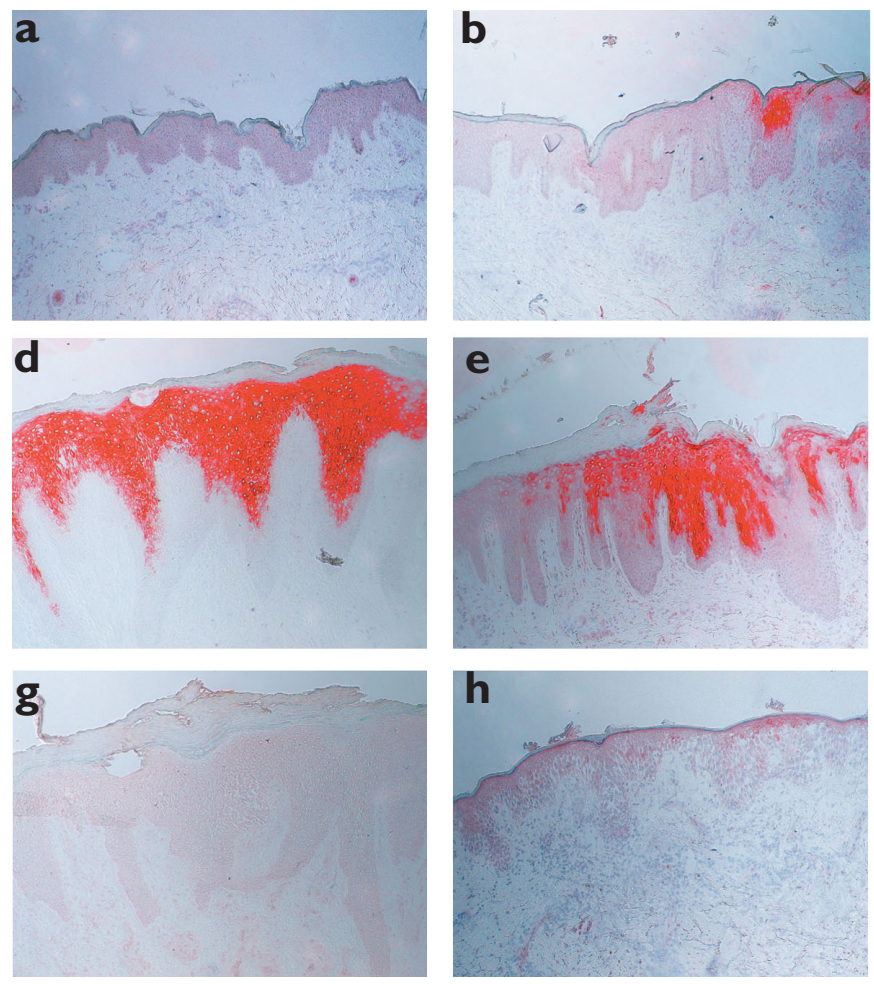

and the $\mathrm{T}_{\mathrm{H}} 1$ cytokine IFN- $\gamma$ were inhibited. These features suggested that TSLP represents a critical mediator in uncontrolled allergic inflammation.

Activation of DCs appears to be a critical step in the pathogenesis of $\mathrm{T}_{\mathrm{H}} 2$-mediated allergic inflammation. Although DCs from allergic individuals preferentially induce a $\mathrm{T}_{\mathrm{H}} 2$-type response ${ }^{32-36}$, the molecular mechanism underlying the signaling of DCs to induce $\mathrm{T}_{\mathrm{H}} 2$ allergic diseases is not understood. Our findings that TSLP is highly expressed by
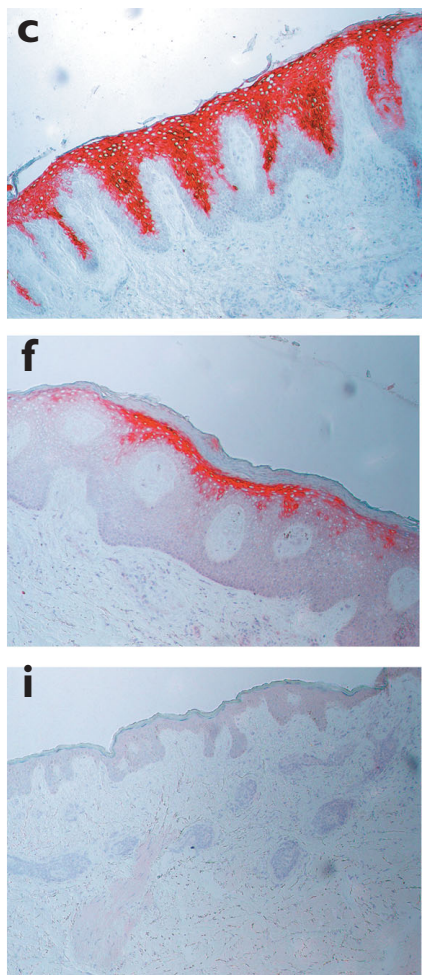

Figure 8. Expression of TSLP in atopic dermatitis. (a) Nonlesional skin from an atopic dermatitis patient. (b,c) Samples from acute atopic dermatitis patients. (d-f) Samples from chronic atopic dermatitis patients. (g) An adjacent section of $\mathbf{d}$, showing negative staining with isotype control. (h) Lesion from a nickel-induced contact allergic dermatitis patient. (i) Lesion from a cutaneous lupus erythematosus patient. TSLP stained red. 
Figure 9. TSLP expression in atopic dermatitis associates with Langerhans cell migration and activation. (a) Double staining of TSLP (red) and DC-LAMP (blue) with a normal skin sample, showing no TSLP expression and no DC-LAMP+ activated DCs within the epidermis or dermis. (b) Double staining of TSLP (red) and langerin (blue), showing the presence of many Langerhans cells within the epidermis. (c) High TSLP expression in atopic dermatitis skin lesion samples, which is associated with the presence of many activated DC-LAMP+ DCs in epidermis and dermis. (d) Many of the DC-LAMP ${ }^{+}$DCs within the dermis in $c$ express langerin.
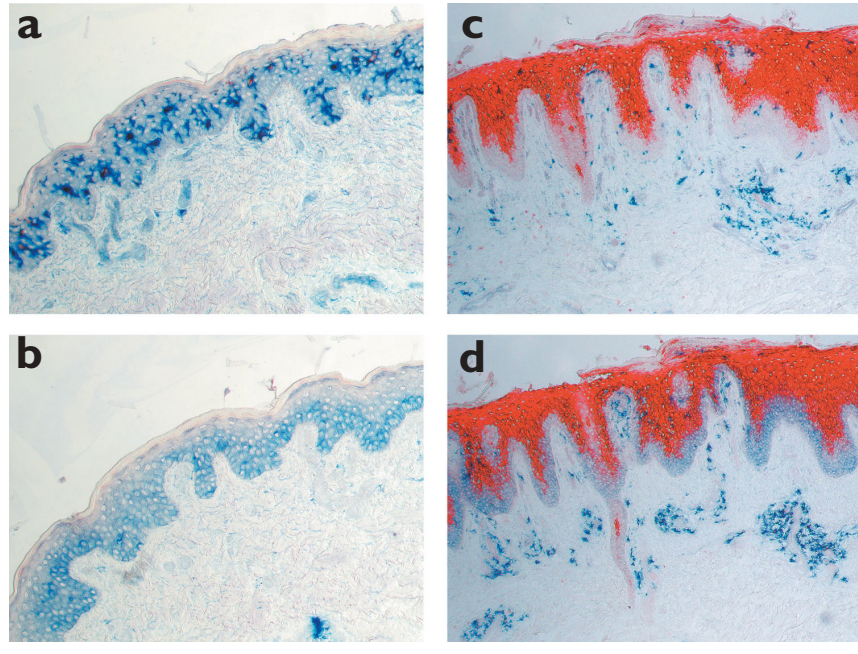

\section{Methods}

DC purification and culture. CD $11 \mathrm{c}^{+} \mathrm{DCs}$ were purified from the adult blood buffy coats of healthy volunteer blood donors (Stanford Medical School Blood Center, Stanford, CA) after separation of peripheral blood mononuclear cells (PBMC) by Ficoll centrifugation and negative depletion of cells that were expressing CD3, CD14, CD19, CD56 and glycophorin A with magnetic beads (Dynal, Oslo, Norway). Depleted cells were further stained with tricolor(TC)-conjugated anti-CD4 (Caltag, Burlingame, CA), phycoerythrin (PE)-antiCD11c, anti-CD3, anti-CD14 and fluorescein isothiocyanate (FITC)-anti-CD16 (Becton Dickinson, Franklin Lakes, NJ). FITC-CD11 $\mathrm{c}^{+}$cells were isolated with a Vantage FACsorter (Becton Dickinson) to reach $>99 \%$ purity. $\mathrm{CD} 11 \mathrm{c}^{+}$DCs were cultured immediately after sorting in RPMI containing $10 \%$ fetal calf serum (FCS), $1 \%$ pyruvate, $1 \%$ HEPES and penicillin-streptomycin. Cells were seeded at $0.5 \times 10^{6} / \mathrm{ml}$ in flat-bottomed 96 -well plates in the presence of TSLP $(15 \mathrm{ng} / \mathrm{ml})$, IL-7 $(50 \mathrm{ng} / \mathrm{ml})$, LPS $(1 \mu \mathrm{g} / \mathrm{ml})$, CD40L-transfected L-fibroblasts $\left(2.5 \times 10^{4} /\right.$ well $)$ or culture medium alone.

DC activation and viability. After $24 \mathrm{~h}$ of culture, DCs were collected and resuspended in an EDTA-containing medium to dissociate the clusters. Viable DCs were first counted with trypan blue exclusion of dead cells. Remaining cells were stained with FITC-conjugated mouse anti-human mAbs that included anti-HLA-DR (Becton Dickinson), anti-CD40, antiCD80 and anti-CD86 (all from Pharmingen, San Diego, CA) and an IgG1 isotype contro (Becton Dickinson); they were analyzed with a FACScan flow cytometer (Becton Dickinson). Dead cells were excluded based on side- and forward-scatter characteristics. For apoptosis detection, cells were stained for 5-10 min with FITC-annexin V (Promega, Madison, WI) and analyzed on a FACScan flow cytometer (Becton Dickinson) without dead-cell exclusion.

DC cytokine production. DC culture supernatants were collected at $24 \mathrm{~h}$, frozen at $-80{ }^{\circ} \mathrm{C}$ and analyzed within 3 months with protein ELISA kits for IL-1 $\beta$, IL-6, IL-12p70, TNF- $\alpha$, TARC, MDC, MIP-1 $\beta$ and RANTES (all from R\&D Systems, Minneapolis, MN).

DC-T cell cocultures. CD11 $\mathrm{c}^{+}$DCs were collected after $24 \mathrm{~h}$ of culture under different conditions, washed twice to remove any cytokine and cocultured with $5 \times 10^{4}$ freshly purified allogeneic naïve $\mathrm{CD} 4{ }^{+} \mathrm{T}$ cells in round-bottomed 96 -well culture plates. Cultures were done in triplicate at increasing DC:T cell ratios. DCs and T cells alone were used as controls. After 5 days, cells were pulsed with $1 \mathrm{mCi}\left[{ }^{3} \mathrm{H}\right]$ thymidine (Amersham Biosciences, Piscataway, NJ) for $16 \mathrm{~h}$ before collecting and counting radioactivity.

T cell cytokine production. After 6 days of coculture, DC-primed CD4 ${ }^{+} \mathrm{T}$ cells were restimulated for $24 \mathrm{~h}$ with plate-bound anti-CD3 $(10 \mu \mathrm{g} / \mathrm{ml})$ and soluble anti-CD28 $(2 \mathrm{ng} / \mathrm{ml})$. Cytokine production was assessed in the culture supernatant by protein ELISA for IL-4, IL-5, IL-10, IL-13, TNF- $\alpha$ and IFN- $\gamma$ (all from R\&D Systems). For intracellular cytokine production, T cells were collected on day 6 of the culture, washed twice and restimulated with PMA + ionomycin in flat-bottomed 96- or 48-well plates at a concentration of $1 \times 10^{6} / \mathrm{ml}$. After $2.5 \mathrm{~h}$, brefeldin A was added at $10 \mathrm{mg} / \mathrm{ml}$. After $5 \mathrm{~h}$, cells were collected, fixed with $2 \%$ formaldehyde, permeabilized with $10 \%$ saponin and stained with PE-conjugated mAbs to IL-4, IL-5, IL-10, IL-13 and TNF- $\alpha$ and FITC-conjugated anti-IFN- $\gamma$ (all from Pharmingen). Stained cells were analyzed on a FACScan flow cytometer (Becton Dickinson).

Quantification of TSLP mRNA in human primary cells. Cryopreserved primary human fibroblasts, epithelial cells, smooth muscle cells, endothelial cells were from Clonetics (Biowhittaker, San Diego, CA), and seeded at 2500-3500 cells $/ \mathrm{cm}^{2}$ in T75 flasks with the appropriate fully supplemented culture medium (Biowhittaker). After two or three passages, cytokines. TSLP may represent a new target to block inflammatory dis eases, in particular allergic diseases. 
cells were collected by light trypsinization and reseeded in 12 or 6 -well plates $\left(5 \times 10^{5}\right.$ cells/well) under different culture conditions, with or without addition of cytokines. After $12-15 \mathrm{~h}$, culture supernatants were collected, adherent cells were lysed, mRNA was extracted with a Qiagen kit (Qiagen, Valencia, CA) and analyzed by real-time quantitative PCR. Mast cells were generated from human $\mathrm{CD} 34^{+}$stem cells cultured with stem cell factor Final culture contained $>95 \% \mathrm{CD} 117^{+} \mathrm{CD} 14^{-} \mathrm{CD} 16^{-}$mast cells. Activation was initiated by cross-linking of the high-affinity IgE receptor with the DX55 mAb (J. Philips, DNAX, Palo Alto, CA). DCs and DC precursors ( $>99 \%$ purity) were isolated by cell-sorting periphera blood cells as described ${ }^{43}$. The following freshly sorted peripheral blood cell subsets, before and after culture, were used for the Taqman-PCR analyses: monoctyes; monocyte-derived immature DCs (imDCs, monocytes cultured for 6 days with GM-CSF and IL-4); monocytederived mature DCs activated by CD40L (CD40L-mDC) or LPS (LPS-mDCs); $\mathrm{CD}^{+}{ }^{+}$Lin ${ }^{-} \mathrm{CD} 11 \mathrm{c}^{-}$plasmacytoid $\mathrm{DC}$ precursors (pDC2); pDC2-derived DCs induced by IL3 (IL-3-DC2) or by HSV-1 (HSV-DC2); CD11 $\mathrm{c}^{+} \mathrm{DCs}\left(\mathrm{CD} 11 \mathrm{c}^{+} \mathrm{DCs}\right)$; CD11 $\mathrm{c}^{+} \mathrm{DCs}$ activated by CD40L (CD40L-CD11 ${ }^{+}$DCs) or by LPS (LPS-CD11 ${ }^{+}$DCs); monocyte-derived macrophages (monocyte cultured for $6 \mathrm{~d}$ with M-CSF); and freshly isolated CD68 ${ }^{+} \mathrm{CD} 16$ neutrophils, CD16 $6^{+} \mathrm{CD} 56^{+} \mathrm{NK}$ cells, CD19+ $\mathrm{B}$ cells and $\mathrm{CD} 3^{+} \mathrm{T}$ cells. For other cell types, cDNA libraries were prepared and used as templates for Taqman-PCR analyses as described ${ }^{17}$. Results are expressed as mRNA levels relative to ubiquitin.

Skin biopsy samples. After obtaining informed consent from patients, $3-6 \mathrm{~mm}$ punch biopsies were taken from either lesional or nonlesional skin from atopic dermatitis $(n=15)$ or disseminated lupus erythematosus patients $(n=5)$ or from normal healthy individuals $(n=$ 11). Patients with a history of allergic contact dermatitis against nickel $(n=8)$ underwent diagnostic nickel patch tests, and skin biopsies (three per patient) were taken before and 6 , 24 or $48 \mathrm{~h}$ after allergen exposure. Skin samples were immediately frozen in liquid nitrogen and stored at $-80^{\circ} \mathrm{C}$. The study was approved by the local ethics committees of the Department of Medicine of the Helsinki-Uusimaa Hospital District, Finland and the Heinrich-Heine University, Dusseldorf, Germany.

Immunohistology. Frozen sections $(8 \mu \mathrm{m})$ of human tonsil or skin were incubated with rat anti-human TSLP (mAb 12F3, DNAX, Palo Alto, CA) at room temperature for $1 \mathrm{~h}$ in PBS. The slides were washed with PBS twice and incubated with biotinylated secondary antibody for $30 \mathrm{~min}$ (PK-4004, Vector Laboratories, Burlingame, CA). The slides were washed an incubated with avidin-peroxidase complex reagents for $30 \mathrm{~min}$ (PK-4004, Vecto Laboratories). The slides were washed and incubated with substrate SK-4200, which stained red (Vector Laboratories). For double staining of human TSLP with DC-LAMP or langerin, the slides were incubated with mouse anti-human DC-LAMP (IM3448) or mouse anti-human langerin (IM3449, Immunotech, Marseille, France) for $1 \mathrm{~h}$; this was followed by red anti-TSLP staining. The slides were washed and incubated with biotinylated anti-mouse Ig (AK-5002, Vector Laboratories), then the avidin-peroxidase complex reagents (AK-5002, Vector Laboratories) for $30 \mathrm{~min}$ each. The slides were washed and the incubated with the substrate SK-5300, which stained blue (Vector Laboratories).

\section{Acknowledgments}

We thank L. Lanier, J.-Z. Chen and J. Banchereau for critical reading of the manuscript and M.Andonian for help with graphics. DNAX Research Institute is supported by ScheringPlough.

\section{Competing interests statement}

The authors declare competing financial interests: see the Nature Immunology website (http://immunology.nature.com) for details.

\section{Received 27 March 2002; accepted I5 May 2002}

I. Kay, A. B. Allergy and allergic diseases. First of two parts. N. Engl. J. Med. 344, 30-37 (2001).

2. Mosmann, T. R. \& Coffman, R. L. $T_{H} I$ and $T_{H} 2$ cells: different patterns of lymphokine secretion lead to different functional properties. Annu. Rev. Immunol. 7, 145-173 (1989).

3. Abbas, A. K., Murphy, K. M. \& Sher, A. Functional diversity of helper T lymphocytes. Nature $\mathbf{3 8 3}$ 787-793 (1996).

4. Constant, S. L. \& Bottomly, K. Induction of $T_{H} I$ and $T_{H} 2 C D 4^{+} T$ cell responses: the alternative approaches. Annu. Rev. Immunol. 15, 297-322 (1997).

5. Renauld, J. C. New insights into the role of cytokines in asthma. J. Clin. Pathol. 54, 577-589 (200I)
6. Holgate, S.T. Science, medicine, and the future. Allergic disorders. Brit. Med.J. 320, 23I-234 (2000). 7. Busse,W.W. \& Lemanske, R. F.Jr. Asthma. N. Engl.J. Med. 344, 350-362 (2001).

8. Banchereau, J. \& Steinman, R. M. Dendritic cells and the control of immunity. Nature 392, 245-252 (1998)

9. Holt, P. G. Macrophage: dendritic cell interaction in regulation of the IgE response in asthma. Clin. Exp. Allergy 23, 4-6 (1993)

10. Lambrecht, B. N. et al. Myeloid dendritic cells induce $\mathrm{T}_{\mathrm{H}} 2$ responses to inhaled antigen, leading to eosinophilic airway inflammation. J. Clin. Invest. 106, 55I-559 (2000).

II. Semper, A. E., Gudin, A. M., Holloway, J. A. \& Holgate, S. T. in Dendritic Cells (eds. Lotze, M. T. \& Thomson, A. W.) 435-456 (Academic Press, San Diego, CA, 1999).

12. Stingl, G. in Immunological and Pharmacological Aspects of Atopic and Contact Eczema (ed. Czernielewski, J.M.) I-9 (Karger, Basel, 199I).

13. Sims, J. E. et al. Molecular cloning and biological characterization of a novel murine lymphoid growth factor. J. Exp. Med. 192, 67I-680 (2000).

14. Tonozuka, Y. et al. Molecular cloning of a human novel type I cytokine receptor related to $\delta / T S L P R$. Cytogenet. Cell Genet. 93, 23-25 (2001).

5. Pandey, A. et al. Cloning of a receptor subunit required for signaling by thymic stromal lymphopoietin. Nature Immunol. I, 59-64 (2000).

16. Park, L. S. et al. Cloning of the murine thymic stromal lymphopoietin (TSLP) receptor: Formation of a functional heteromeric complex requires interleukin 7 receptor. J. Exp. Med. 192, 659-670 (2000).

17. Reche, P.A. et al. Human thymic stromal lymphopoietin preferentially stimulates myeloid cells. J. Immunol. 167, 336-343 (200I).

18. Ray, R. J., Furlonger, C., Williams, D. E. \& Paige, C. J. Characterization of thymic stromal-derived lymphopoietin (TSLP) in murine B cell development in vitro. Eur. J. Immunol. 26, 10-16 (1996).

19. Levin, S. D. et al. Thymic stromal lymphopoietin: a cytokine that promotes the development of $\lg \mathrm{M}^{+} \mathrm{B}$ cells in vitro and signals via a novel mechanism.J. Immunol. 162, 677-683 (1999).

20. Koch, F. et al. High level IL-12 production by murine dendritic cells: upregulation via MHC class II and CD40 molecules and downregulation by IL-4 and IL-10.J. Exp. Med. 184, 74I-746 (1996).

21. Cella, M. et al. Ligation of CD40 on dendritic cells triggers production of high levels of interleukin-12 and enhances T cell stimulatory capacity:T-T help via APC activation. I. Exp. Med 184, 747-752 (1996).

22. Pulendran $B$ et al Distinct dendritic cell subsets differentially regulate the class of immune response in vivo. Proc. Natl. Acad. Sci. USA 96, 1036-104I (1999).

23. Maldonado-Lopez, R. et al. CD $8 \alpha^{+}$and $C D 8 \alpha^{-}$subclasses of dendritic cells direct the development of distinct T helper cells in vivo.J. Exp. Med. 189, 587-592 (1999).

24. Rissoan, M. C. et al. Reciprocal control of $T$ helper cell and dendritic cell differentiation. Science $\mathbf{2 8 3}$ II83-1186 (1999)

25. Homey, B. \& Zlotnik, A. Chemokines in allergy. Curr. Opin. Immunol. I I, 626-634 (1999).

26. Moore, K.W., de Waal Malefyt, R., Coffman, R. L. \& O'Garra, A. Interleukin- 10 and the interleukin- 10 receptor. Annu. Rev. Immunol. 19, 683-765 (200I).

27. O'Garra, A. Cytokines induce the development of functionally heterogeneous $T$ helper cell subsets. Immunity 8, 275-283 (1998).

28. Vestergaard, $C$. et al. A TH2 chemokine, TARC, produced by keratinocytes may recruit CLA+CCR4 lymphocytes into lesional atopic dermatitis skin. J. Invest. Dermatol. I I 5, 640-646 (2000)

29. Imai, T. et al. Selective recruitment of CCR4-bearing $T_{H} 2$ cells toward antigen-presenting cells by the $\mathrm{CC}$ chemokines thymus and activation-regulated chemokine and macrophage-derived chemokine. Int. Immunol. II, 8I-88 (1999).

30. Andrew, D. P. et al. C-C chemokine receptor 4 expression defines a major subset of circulating non-

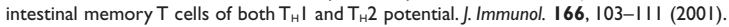

31. de Saint-Vis, B. et al. A novel lysosome-associated membrane glycoprotein, DC-LAMP, induced upon DC maturation, is transiently expressed in MHC class II compartment. Immunity 9, 325-336 (1998).

32. Hammad, $\mathrm{H}$. et al. $\mathrm{T}_{\mathrm{H}} 2$ polarization by Derp I-pulsed monocyte-derived dendritic cells is due to the allergic status of the donors. Blood 98, II35-1141 (2001).

33. McWilliam, A. S. et al. Dendritic cells are recruited into the airway epithelium during the inflammatory response to a broad spectrum of stimuli.J. Exp. Med. 184, 2429-2432 (1996).

34. Novak, N., Haberstok, J., Geiger, E. \& Bieber, T. Dendritic cells in allergy. Allergy 54, 792-803 (1999).

35. Tunon-De-Lara, J. M. et al. Dendritic cells in normal and asthmatic airways: expression of the $\alpha$ subunit of the high affinity immunoglobulin E receptor ( $\mathrm{Fc \varepsilon} \mathrm{Rl}-\alpha$ ). Clin. Exp. Allergy 26, 648-655 (1996).

36. Holt P. G. Macaubas, C Cooper D. Nelson, D. I \& McWilliam, A. S. Th-1/Th-2 switch regulation in immune responses to inhaled antigens. Role of dendritic cells in the aetiology of allergic respiratory disease. Adv. Exp. Med. Biol. 417, 301-306 (1997).

37. Homey B. et al. CCL27-CCRIO interactions regulate T cell-mediated skin inflammation. Nature Med. 8, 157-65 (2002).

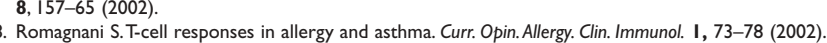

39. Robinson, D. S. et al. Increased interleukin-10 messenger RNA expression in atopic allergy and asthma. Am.J. Respir. Cell. Mol. Biol. 14, I I3-I I7 (1996).

40. Borish, L. et al. Interleukin-10 regulation in normal subjects and patients with asthma.J.Allergy. Clin. Immunol. 97, 1288-1296 (1996).

4I. Akbari, O., DeKruyff, R. H. \& Umetsu, D.T. Pulmonary dendritic cells producing IL-10 mediate tolerance induced by respiratory exposure to antigen. Nature Immunol. 2,725-73I (200I).

42. Zuany-Amorim, C. et al. Interleukin-10 inhibits antigen-induced cellular recruitment into the airways of sensitized mice. J. Clin. Invest. 95, 2644-265I (1995).

43. Siegal, F. P. et al. The nature of the principal type I interferon-producing cells in human blood. Science 284, $1835-1837$ (1999). 